\title{
Prediction of Aphasia Outcome Using Diffusion Tensor Tractography for Arcuate Fasciculus in Stroke
}

S.H. Kim and S.H. Jang

\begin{abstract}
BACKGROUND AND PURPOSE: The AF is an important neural tract in language function. We investigated aphasia outcome according to DTT findings for $\mathrm{AF}$ in the early stage of stroke.

MATERIALS AND METHODS: Twenty-five consecutive patients with aphasia and stroke and 12 control subjects were recruited. The AQ of K-WAB was used for the assessment of aphasia in the early stage of stroke (10-30 days) and at approximately 6 months after onset. We classified the patients into 3 groups according to the severity of left AF injury: type A, the AF was not reconstructed; type B, the AF was disrupted; and type $\mathrm{C}$, the AF was preserved around the lesion.
\end{abstract}

RESULTS: When comparing AQ among AF types at early evaluation, the type $\mathrm{C}$ score $(32.84 \pm 18.05)$ was significantly higher than type $\mathrm{A}$ $(3.60 \pm 2.73)(P<.05)$. However, no significant difference was observed between types $A$ and $B(18.02 \pm 17.19)$ or between types $B$ and $C(P>$ .05). At late evaluation, the $A Q$ values of types $B(52.43 \pm 25.75)$ and $C(68.08 \pm 15.76)$ were higher than that of type $A(10.98 \pm 3.90)(P<$ .05). However, there was no significant difference between types $B$ and $C$.

CONCLUSIONS: The aphasia outcome of the patients whose left AF could be reconstructed was better than that in patients whose left AF could not be reconstructed, irrespective of its integrity. We believe that evaluation of the left AF by using DTT in the early stage of stroke could be helpful in predicting aphasia outcome in patients with stroke.

ABBREVIATIONS: AF = arcuate fasciculus; $A Q$ = aphasia quotient; DTI = diffusion tensor imaging; DTT = diffusion tensor tractography; FA = fractional anisotropy; $\mathrm{K}-\mathrm{WAB}=$ Korean-Western Aphasia Battery

S troke is a leading cause of major adult disability, and aphasia is one of the most common consequences of stroke. It has been reported that $21 \%-38 \%$ of patients with stroke have aphasia in the acute stage. ${ }^{1-3}$ Recovery from aphasia occurs mainly during the first 3 months after stroke; finally, $10 \%-18 \%$ of patients with stroke have aphasia in the chronic stage. ${ }^{2,4,5}$ Therefore, it is important to predict the prognosis of aphasia at an early stage in patients with stroke because it could provide useful information for planning specific rehabilitation strategies and for estimating the duration of rehabilitation. Previously, many studies have attempted to predict the out-

Received April 25, 2012; accepted after revision June 21.

From the Department of Physical Medicine and Rehabilitation, College of Medicine, Yeungnam University, Taegu, Republic of Korea.

This work was supported by the DGIST R\&D Program of the Ministry of Education, Science and Technology of Korea (11-IT-01).

Please address correspondence to Sung Ho Jang, MD, Department of Physical Medicine and Rehabilitation, College of Medicine, Yeungnam University 317-1, Daemyungdong, Namku, Taegu, 705-717, Republic of Korea; e-mail: strokerehab@ hanmail.net

-- Indicates open access to non-subscribers at www.ajnr.org

http://dx.doi.org/10.3174/ajnr.A3259 come of stroke-related aphasia by using brain CT, conventional brain MR imaging, and functional neuroimaging. ${ }^{6-8}$ However, these neuroimaging modalities are limited in that they cannot reconstruct and estimate neural tracts. In contrast, DTT, a 3D visualized version of DTI, allows 3D visualization of the architecture and integrity of neural tracts at the subcortical level. ${ }^{9,10}$ DTT for the neural tract has been used for prediction of outcome for the corresponding function. For example, many studies have demonstrated the predictive value of DTT of the corticospinal tract for evaluating the motor outcome in patients with stroke. ${ }^{11-13}$ However, little is known about the usefulness of DTT for the AF, which is the important neural tract for language, connecting Wernicke and Broca areas, in predicting the aphasia outcome in patients with stroke, though few studies have demonstrated the clinical usefulness of DTT for AF. ${ }^{14-16}$

In the current study, we investigated aphasia outcome according to DTT findings for AF in early-stage stroke.

\section{MATERIALS AND METHODS \\ Patients}

Twenty-five right-handed patients with aphasia (16 men, 9 women; mean age, 57.3 years; range, 33-74 years; cerebral infarct, 12 
patients; intracerebral hemorrhage, 13 patients) and 12 age- and sex-matched right-handed healthy control subjects ( 8 men, 4 women; mean age, 56.3 years; range, $30-76$ years) with no history of neurologic or psychiatric disease were recruited for this study. The twenty-five patients were recruited consecutively among 543 patients with stroke admitted to the department of rehabilitation from January 2009 to May 2011, according to the following inclusion criteria: 1) first-ever stroke: no previous history of cerebral ischemia or hemorrhage; 2) age range, 20 75 years; 3) stroke lesion located at the left basal ganglia or/and corona radiata level; 4) aphasia, AQ score on K-WAB below $92.8^{17}$; 5) presence of left AF injury defined when the FA value of the left AF deviated more than 2 SDs from the value of the control group or the integrity of the left AF was disrupted; 6) DTI scanning and early language evaluation performed within 30 days of stroke onset, and late language evaluation performed approximately 6 months after stroke onset; and 7) no history of head trauma or psychiatric disorder. This study was performed retrospectively, and the study protocol was approved by the institutional review board of our hospital.

\section{Language Evaluation}

The AQ of K-WAB, which is the Korean version of the Western Aphasia Battery, was used for assessment of language dysfunction (range, $0 \sim 100$ th percentile); a higher score means better function. ${ }^{17}$ It was performed at an early stage of stroke (mean, 20.6 days after onset; range, 10-30 days) and approximately 6 months after onset (mean follow-up period, 171.5 days after onset; range, 93-354 days; 93-180 days, 14 patients; 180-270 days, 9 patients; 270-354 days, 2 patients) because most recovery has been known to be achieved during the first 3-6 months after onset. ${ }^{18-22}$ Both the reliability and validity of the K-WAB have been well established previously. ${ }^{17,23}$

\section{Diffusion Tensor Imaging and Tractography}

DTI data were acquired at an average of 19.6 days after onset (range, 9-30 days) by using the 1.5T Gyroscan Intera system (Philips Healthcare, Best, the Netherlands), equipped with a Synergy-L sensitivity encoding (SENSE) head coil (Philips Healthcare) and using a single-shot, spin-echo-planar imaging pulse sequence. Sixty-seven contiguous sections were acquired for each of the 32 noncollinear diffusion-sensitizing gradients. Imaging parameters were as follows: acquisition matrix $=96 \times 96$, reconstructed to a $128 \times 128$ matrix; FOV $=221 \times 221 \mathrm{~mm}^{2}$; $\mathrm{TR}=$ $10,726 \mathrm{~ms}$; TE $=76 \mathrm{~ms}$; parallel imaging reduction factor (SENSE factor) $=2$; EPI factor $=49$ and $b=1000 \mathrm{~s} / \mathrm{mm}^{2} ; \mathrm{NEX}=1$; and section thickness of $2.3 \mathrm{~mm}$ (acquired isotropic voxel size, $2.3 \times$ $2.3 \times 2.3 \mathrm{~mm}^{3}$ ).

Eddy current-induced image distortions were removed by using affine multiscale $2 \mathrm{D}$ registration at the Oxford Centre for FMRIB Software Library (FSL; www.fmrib.ox.ac.uk/fsl). ${ }^{24}$ DTI Studio software (CMRM, Johns Hopkins Medical Institute, Baltimore, Maryland) ${ }^{25}$ was used for evaluation of the left AF, and it was based on the fiber assignment continuous tracking algorithm and the multiple-ROIs approach. Using the method of Nucifora et al and Vernooij et al, ${ }^{26,27}$ we selected 2 ROIs for tracking of the $\mathrm{AF}$ (ie, the seed region of interest in the posterior parietal area of the superior longitudinal fascicle and the target region of interest in the posterior temporal lobe). Termination criteria used for fiber tracking were FA $<0.2$ and angle $<60$. We measured the value of the FA and ADC of the left AF for DTI parameters. We classified the patients into 3 groups according to the severity of the left AF injury: type A, the AF not reconstructed due to severe injury. We confirmed the nonreconstruction of the AF by lowering the AF value to 0.1 and giving only 1 region of interest along the AF pathway. In addition, we confirmed severe degeneration of the AF on the color map: In type B, the AF was disrupted between Wernicke and Broca areas; and in type $\mathrm{C}$, the AF was preserved around the stroke lesion (ie, the tract originating from the Wernicke area and passing around the lesion to the Broca area, Fig 1).

\section{Statistical Analysis}

All statistical analyses were performed by using the Statistical Package for the Social Sciences for Windows (SPSS, Version 12.0K; SPSS, Seoul, Korea). One-way ANOVA analysis was performed for determining significant differences in FA and ADC values of the $\mathrm{AF}$ among the 3 types of patient groups and the control group and AQ values at early and late evaluation among the 3 patient groups. In addition, we compared the differences of age, sex, DTI scanning time from onset, and the times to K-WAB evaluations in the 3 types of patient groups by using 1-way ANOVA analysis. The Pearson correlation analysis was used to estimate the significant correlations between FA values of the left $\mathrm{AF}$ and the $\mathrm{AQ}$ values, repetition scores, time intervals of language assessments, and AQs at late evaluation. Furthermore, linear regression analysis was performed to determine the effect of the initial AQ in the prediction of aphasia recovery. The level of statistical significance was set at $P<.05$.

\section{RESULTS}

Four patients ( 1 man; mean age, 71 years; range, 66-74 years) belonged to type A, 11 patients belonged to type B ( 6 men; mean age, 57 years; range, $40-73$ years); and 10 patients, to type $C$ ( 7 men; mean age, 53 years; range, $33-66$ years). No significant differences were found among the groups in the time to DTI scanning after onset (type A: mean, 22 days; range, 15-30; type B: mean, 20 days; range, 9-30 days; type $C$ : mean, 18 days; range, $11-29$ days; type A-B: $P=.582$; type A-C: $P=.392$; type $\mathrm{B}-\mathrm{C}: P=$ .667 ) and sex (type A-B: $P=.326$; type A-C: $P=.145$; type B-C: $P=.490$ ). The times to early (type A: mean, 22 days; range, 14-30 days; type B: mean, 21 days; range, 13-30 days; type C: mean, 19 days; range, $10-29$ days; type A-B: $P=.893$; type A-C: $P=.483$; type B-C: $P=.441$ ) and late K-WAB evaluations (type A: mean, 157 days; range, 93-232 days; type B: mean, 156 days; range, 105 249 days; type C: mean, 194 days; range, 105-354 days; type A-B: $P=.981$; type A-C: $P=.334$; type B-C: $P=.185$ ) were not significantly different among the 3 groups. Moreover, the time interval of early and late language assessments was not significantly associated with AQ at late evaluation $(r=0.029, P=.891)$. However, age was increased in type $\mathrm{A}$, compared with the other groups (type A-B: $P=.041$; type A-C: $P=.012$; type B-C: $P=$ $.424)$.

In types $\mathrm{B}$ and $\mathrm{C}$ of the patient groups, the $\mathrm{FA}$ and $\mathrm{ADC}$ values of the left AF showed differences in comparison with those of 

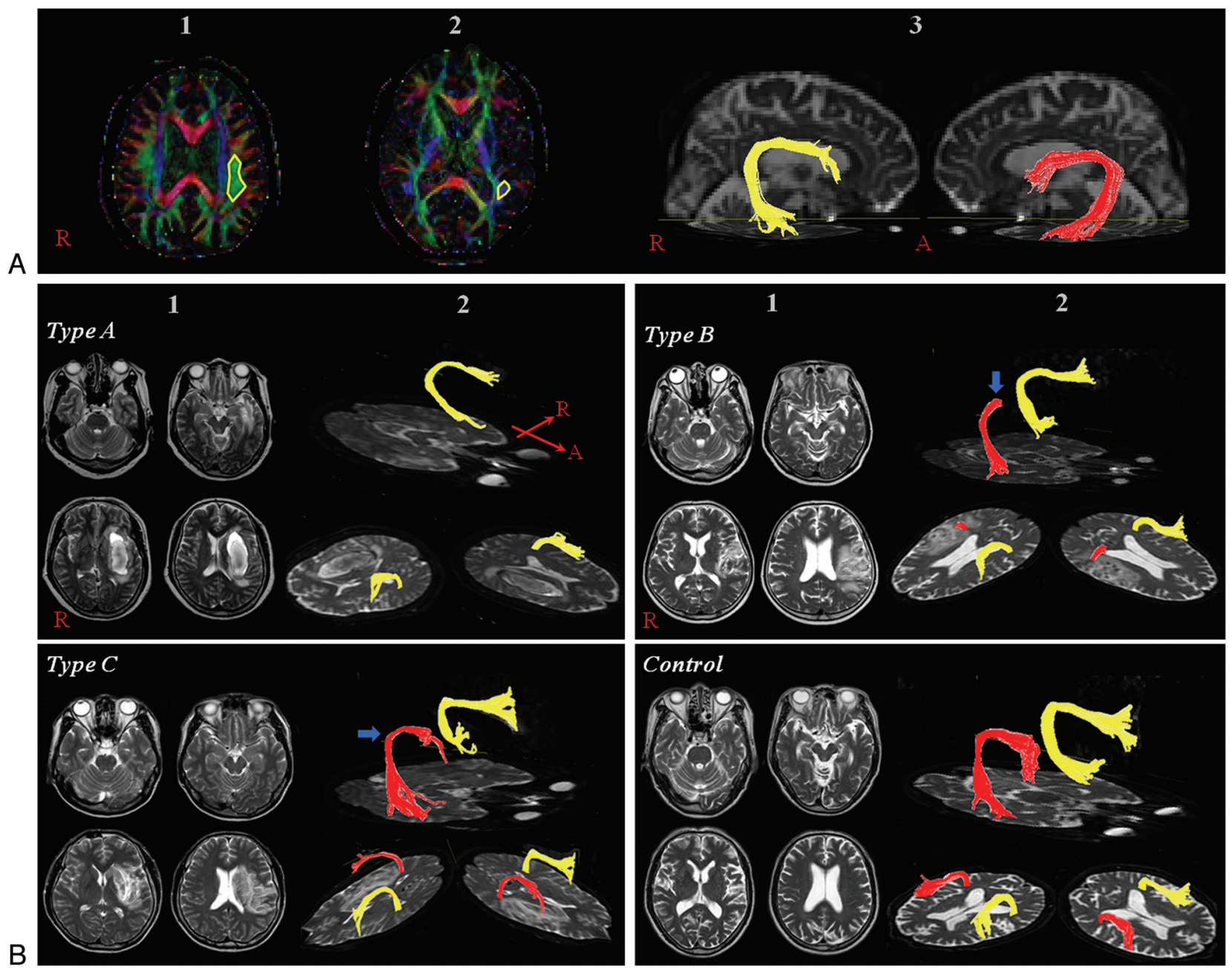

FIG 1. A, 1) Seed region of interest, 2) target region of interest, and 3) reconstructed AF. B, 1) T2-weighted brain MR imaging in subacute stroke shows brain lesions in the left corona radiata and at the basal ganglia level, and 2) DTT for the AF (right AF, yellow; left AF, red). The blue arrow indicates disruption of the left AF around the stroke lesion.

Table 1: Diffusion tensor imaging data of the arcuate fasciculus in the patient and control groups ${ }^{\mathrm{a}}$

\begin{tabular}{lccccc}
\hline & \multicolumn{2}{c}{ Left } & & \multicolumn{2}{c}{ Right } \\
\cline { 2 - 3 } \cline { 5 - 6 } Type & FA & ADC & & FA & ADC \\
\hline A & - & - & & $0.4445(0.0197)$ & $0.8100(0.0491)$ \\
B & $0.3934(0.0442)^{\mathrm{b}}$ & $0.8681(0.0787)^{\mathrm{b}}$ & & $0.4538(0.0264)$ & $0.7952(0.0331)$ \\
C & $0.4103(0.0324)^{\mathrm{b}}$ & $0.8344(0.0998)^{\mathrm{b}}$ & & $0.4801(0.0336)$ & $0.7775(0.0347)$ \\
Control & $0.5054(0.0189)$ & $0.7392(0.0296)$ & & $0.4754(0.0269)$ & $0.7748(0.0220)$ \\
\hline
\end{tabular}

${ }^{a}$ The values are mean (SD).

${ }^{\mathrm{b}}$ Statistically different compared with the control group $(P<.05)$.

Table 2: AQ of K-WAB at the time of early and late evaluation ${ }^{\mathrm{a}}$

\begin{tabular}{ccccc}
\hline Evaluation & Type A & Type B & Type C & P Value \\
\hline Early & $3.60(2.73)$ & $18.02(17.19)$ & $32.84(18.05)$ & \\
A-B & & & & .146 \\
A-C & & & & .006 \\
B-C & & & .050 \\
Late & $10.98(3.90)$ & $52.43(25.75)$ & $68.08(15.76)$ & \\
A-B & & & & .002 \\
A-C & & & & .000 \\
B-C & & & .089 \\
\hline
\end{tabular}

${ }^{a}$ The values are mean (SD). controls $(P<.05)$; however, there was no difference between types $\mathrm{B}$ and $\mathrm{C}$ (FA: $P>$ .05 ; ADC: $P>.05$ ) (Table 1). In the right $\mathrm{AF}$, we could not observe any differences in terms of FA and ADC values between each type of patient group and the control group $(P>.05)$.

When comparing AQs among AF types at the early evaluation, type C (32.84 \pm 18.05) showed a significantly higher mean score than type A $(3.60 \pm 2.73 ; P<.05)$ (Table 2). However, no significant difference was observed between types A and B (18.02 \pm 17.19$)$ or between types B and C. At the late evaluation, the AQ values of types $B(52.43 \pm 25.75)$ and $C(68.08 \pm 15.76)$ were higher than that of type A $(10.98 \pm 3.90)(P<.05)$. However, we could not observe significant difference between types $B$ and $C$, though the AQ of type $C$ was higher than that of type $B$.

The FA value of the left AF in types B and C showed correlation with the AQ at late evaluation (AQ at late evaluation, $r=0.605$, $P=.004$ ) (Fig 2). However, the AQ at early evaluation and the repetition score of the AQ at early and late evaluations did not 

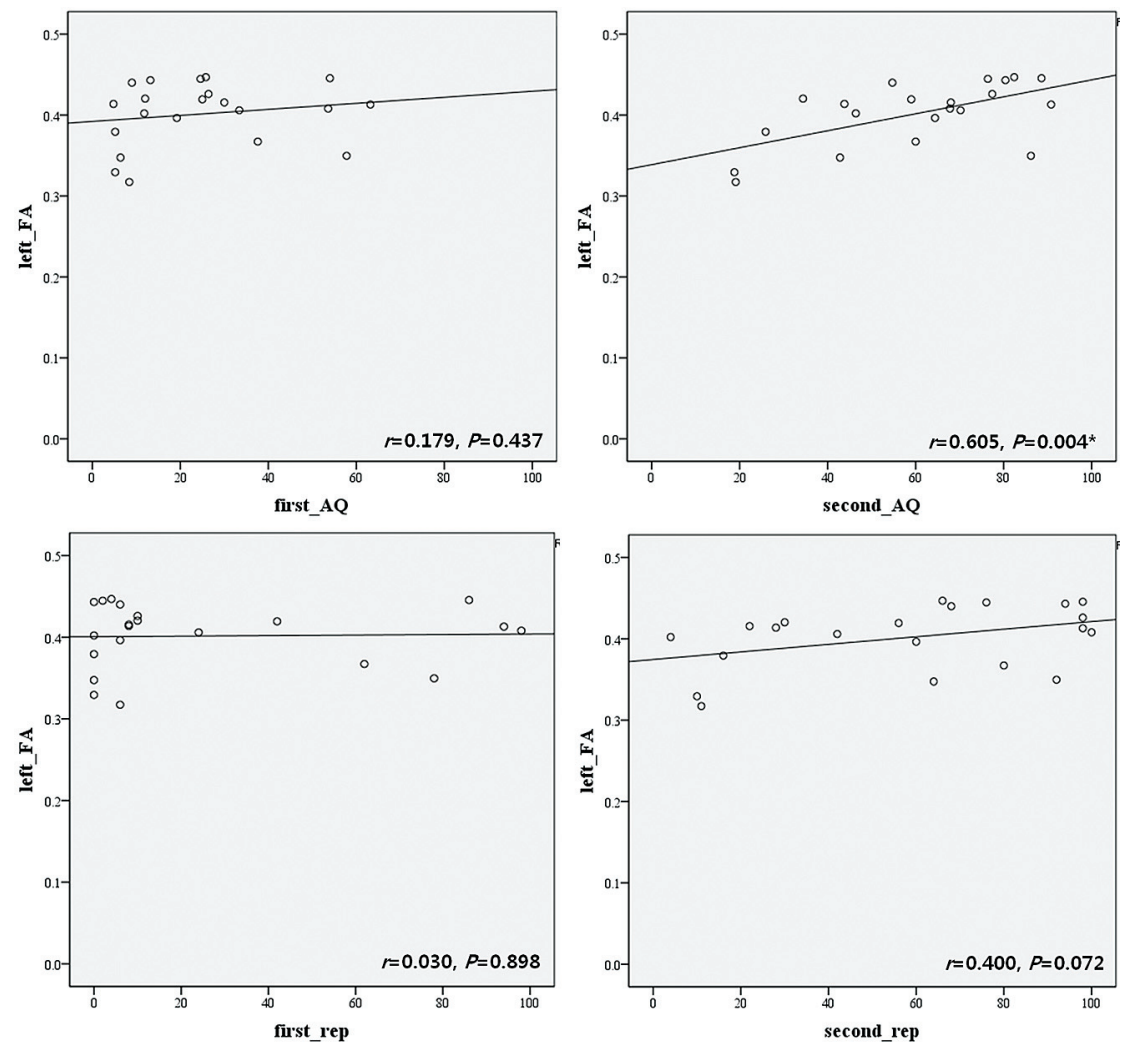

FIG 2. The correlation between the FA of the left $A F$ and the $A Q$ repetition score. rep indicates repetition; the asterisk, $P<.05$.

show correlation with the FA value of the left AF (AQ at early evaluation, $r=0.179, P=.437$; repetition of AQ at early evaluation, $r=0.030, P=.898$; repetition of AQ at late evaluation, $r=$ $0.400, P=.072$ ). In addition, the score of the AQ at early evaluation was an independent variance in the prediction of the AQ at late evaluation $(P=.000)$.

\section{DISCUSSION}

In this study, we tried to clarify the aphasia outcome according to the integrity of the left AF in patients with stroke and found the following results: 1 ) in type $B$ and $C$ patients, the $F A$ values of the left AF were decreased and the ADC values were increased, compared with those of controls; 2) at late K-WAB evaluation, the mean values of AQs in patient groups were 10.98 (type A), 52.43 (type B), and 68.08 (type C); and 3) at late K-WAB evaluation, the AQs of types B and C were better than that of type A, and there was no significant difference between type B and type C.

The FA value represents the degree of directionality of microstructures (eg, axons, myelin, and microtubules), ${ }^{9,28}$ and the ADC value indicates the magnitude of water diffusion, ${ }^{9,28}$ which can increase with some forms of pathology, particularly vasogenic edema or accumulation of cellular debris from axonal damage. Therefore, decrease of FA values and an increase of ADC values may indicate injury of the neural tract. Disruption of the DTT integrity for a neural tract also appears to indicate injury of the neural tract. Taken together, our results showing decreased FA values and increased $\mathrm{ADC}$ values of types $\mathrm{B}$ and $\mathrm{C}$, disruption of the left AF in type B, and nonreconstruction of the left AF in type A appeared to indicate neural injury of the left AF. It seemed that the nonreconstruction of the left AF could be ascribed to the severe injury of the $\mathrm{AF}$ and/or Wallerian degeneration following severe injury of the left $\mathrm{AF}^{29}$ Furthermore, we found a positive correlation between the FA value of the left $\mathrm{AF}$ and the AQ scores and repetition of the AQ. These results seem to support the previous studies about the role of AF in language function: Injury of the AF showed various language dysfunction as well as conduction aphasia. $^{29-32}$

The early AQ score was found to be correlated with the late $\mathrm{AQ}$ in regression analysis. These results coincided with the results of previous studies finding that the severity of aphasia at the acute stage is a significant predictor of aphasia outcome..$^{2,21,33-35} \mathrm{Be}$ sides, at late AQ evaluation, the AQs of types $\mathrm{B}$ and $\mathrm{C}$ were better than that of type $\mathrm{A}$, and there was no significant difference between types B and type C. These results indicated that patients with stroke in whom the left AF could not be reconstructed due to its severe injury showed worse prognosis; in contrast, patients with stroke whose left $\mathrm{AF}$ could be reconstructed revealed better prognosis, irrespective of the integrity of the left AF.

In addition, we suggest several reasons for the lack of significant difference between types B and C at late AQ evaluation. First, the disrupted $\mathrm{AF}$ at the early stage of stroke could show recovery until the late AQ evaluation. However, we could not confirm this because we did not obtain the follow-up DTI. According to the previous studies on the recovery mechanism of aphasia, we can assume several other ways of recovery: recovery via other neural tracts such as the superior longitudinal fasciculus, contribution of the unaffected (right) hemisphere, or interhemispheric dissociated lateralization of the Broca and Wernicke areas (Broca and Wernicke areas are located in different hemispheres)., ${ }^{70,36-38}$ Second, it has been reported that the typical type of the AF injury was conduction aphasia, and the prognosis of conduction aphasia was usually good. Kertesz and $\mathrm{McCabe}^{39}$ reported that 5 (62.5\%) of 8 patients with conduction aphasia were completely recovered. ${ }^{30,40}$ Moreover, several studies suggested that the AF was not necessary for speech repetition. ${ }^{31,32,36-41}$ Third, another possibility might be the small number of subjects. Therefore, we believe further DTI follow-up studies involving a large number of subjects are necessary to clarify this issue.

Several studies have reported on the usefulness of DTT for the AF in patients with stroke. ${ }^{14-16,32}$ In 2008, Breier et al ${ }^{16}$ compared the Western Aphasia Battery AQ (repetition, naming, and comprehension) with $\mathrm{FA}$ values of the $\mathrm{AF}$ and superior longitudinal fasciculus in 20 patients with stroke with left hemispheric lesions and found that the FA value of the left AF on DTT, which was scanned at the chronic stage (1-72 months), was correlated with repetition AQ. ${ }^{16}$ Subsequently, Schlaug et al in $2009^{15}$ demon- 
strated that the number of fibers and volume of the right AF were increased after 75-80 intonation-based speech therapy sessions in 6 patients with chronic stroke with Broca aphasia. ${ }^{15}$ Recently, Kim et al in $2011^{32}$ reported that DTT for the AF could provide useful information on the presence or severity of injury of the AF, which could not be detected on conventional brain MR imaging in 5 patients with stroke.

On the other hand, to the best of our knowledge, only 1 previous study used DTI for predicting the outcome of aphasia in patients with stroke. In 2009, Hosomi et al ${ }^{14}$ measured and compared the asymmetry of FA and the number of fibers between the right and left AF on DTT obtained within 2 days after onset in 13 patients with left middle cerebral artery infarcts; loss of leftward asymmetry in fiber numbers of AF predicted aphasia at discharge (13-52 days after onset). In contrast, in the current study, we performed DTI in the early stage of stroke because DTI parameters could be unstable at acute and subacute stages (onset, 5 days) of stroke, ${ }^{42,43}$ and we tried to get outcome data approximately 6 months after stroke onset because the recovery of aphasia occurs mainly during the first 3-6 months after stroke. ${ }^{22,24,35}$ Recently, Marchina et al in $2011^{8}$ estimated the volume of 3 language-related neural tracts (the AF, extreme capsule, and uncinate fasciculus, which were affected by a stroke lesion). They found that lesion loads of the AF were predictive of language function in terms of rate, level of information provided, efficacy of speech, and naming ability in 23 patients with chronic aphasia. This lesion-load method for the AF can be applied at the early stage of stroke for prognosis prediction as in the current study.

The main limitation of our study was the small number of patients. Further studies involving larger numbers of subjects are warranted. In addition, we believe that follow-up DTI studies are also needed. With regard to the fiber-tracking method in DTI, the deterministic method has lower sensitivity in the clarification of crossing fibers than the probabilistic method. ${ }^{44}$ However, the deterministic method is highly beneficial for reproducible reconstruction of prominent white matter tracts. ${ }^{45}$

\section{CONCLUSIONS}

In the current study, we tried to predict the aphasia outcome according to the DTT finding of the left AF in patients with stroke. We found that after approximately 6 months from stroke onset, the average AQ values were 10.98 (in patients whose left AF could not be reconstructed), 52.43 (in patients who showed disruptions of the left AF), and 68.08 (in patients who showed intact integrity of the left AF). The aphasia outcome of the patients whose left AF could be reconstructed was better than that in patients whose left AF could not be reconstructed, irrespective of the left AF integrity. Consequently, we believe that evaluation of the left AF by using DTT at the early stage of stroke would be helpful in predicting aphasia outcome in patients with stroke.

\section{REFERENCES}

1. Engelter ST, Gostynski M, Papa S, et al. Epidemiology of aphasia attributable to first ischemic stroke: incidence, severity, fluency, etiology, and thrombolysis. Stroke 2006;37:1379-84

2. Wade DT, Hewer RL, David RM, et al. Aphasia after stroke: natural history and associated deficits. J Neurol Neurosurg Psychiatry 1986;49:11-16
3. Kauhanen ML, Korpelainen JT, Hiltunen P, et al. Aphasia, depression, and non-verbal cognitive impairment in ischaemic stroke. Cerebrovasc Dis 2000;10:455-61

4. Robey RR. A meta-analysis of clinical outcomes in the treatment of aphasia. J Speech Lang Hear Res 1998;41:172-87

5. Berthier ML. Poststroke aphasia: epidemiology, pathophysiology and treatment. Drugs Aging 2005;22:163-82

6. Payabvash S, Kamalian S, Fung S, et al. Predicting language improvement in acute stroke patients presenting with aphasia: a multivariate logistic model using location-weighted atlas-based analysis of admission CT perfusion scans. AJNR Am J Neuroradiol 2010;31: $1661-68$

7. Thulborn KR, Carpenter PA, Just MA. Plasticity of language-related brain function during recovery from stroke. Stroke 1999;30:749-54

8. Marchina S, Zhu LL, Norton A, et al. Impairment of speech production predicted by lesion load of the left arcuate fasciculus. Stroke 2011;42:2251-56

9. Assaf Y, Pasternak O. Diffusion tensor imaging (DTI)-based white matter mapping in brain research: a review. $J$ Mol Neurosci 2008;34:51-61

10. Mori S, Crain BJ, Chacko VP, et al. Three-dimensional tracking of axonal projections in the brain by magnetic resonance imaging. Ann Neurol 1999;45:265-69

11. Jang SH. Prediction of motor outcome for hemiparetic stroke patients using diffusion tensor imaging: A review. NeuroRehabilitation 2010;27:367-72

12. Puig J, Pedraza S, Blasco G, et al. Acute damage to the posterior limb of the internal capsule on diffusion tensor tractography as an early imaging predictor of motor outcome after stroke. AJNR Am J Neuroradiol 2011;32:857-63

13. Kwon YH, Son SM, Lee J, et al. Combined study of transcranial magnetic stimulation and diffusion tensor tractography for prediction of motor outcome in patients with corona radiata infarct. $J$ Rehabil Med 2011;43:430-34

14. Hosomi A, Nagakane Y, Yamada K, et al. Assessment of arcuate fasciculus with diffusion-tensor tractography may predict the prognosis of aphasia in patients with left middle cerebral artery infarcts. Neuroradiology 2009;51:549-55

15. Schlaug G, Marchina S, Norton A. Evidence for plasticity in whitematter tracts of patients with chronic Broca's aphasia undergoing intense intonation-based speech therapy. Ann N Y Acad Sci 2009;1169:385-94

16. Breier JI, Hasan KM, Zhang W, et al. Language dysfunction after stroke and damage to white matter tracts evaluated using diffusion tensor imaging. AJNR Am J Neuroradiol 2008;29:483-87

17. Kim H, Na DL. Normative data on the Korean version of the Western Aphasia Battery. J Clin Exp Neuropsychol 2004;26:1011-20

18. Ferro JM, Mariano G, Maduriera S. Recovery from aphasia and neglect. Cerebrovasc Dis 1999;9(suppl 5):6-22

19. Demeurisse G, Demol O, Derouck M, et al. Quantitative study of the rate of recovery from aphasia due to ischemic stroke. Stroke 1980;11:455-58

20. Sarno MT, Levita E. Natural course of recovery in severe aphasia. Arch Phys Med Rehabil 1971;52:175-78

21. Pedersen PM, Jørgensen HS, Nakayama H, et al. Aphasia in acute stroke: incidence, determinants and recovery. Ann Neurol 1995;38:659-66

22. Hartman J. Measurement of early spontaneous recovery from aphasia with stroke. Ann Neurol 1981;9:89-91

23. Shewan CM, Kertesz A. Reliability and validity characteristics of the Western Aphasia Battery (WAB). J Speech Hear Disord 1980;45: 308-24

24. Smith SM, Jenkinson M, Woolrich MW, et al. Advances in functional and structural MR image analysis and implementation as FSL. Neuroimage 2004;23(suppl 1):S208-19

25. Jiang H, van Zijl PC, Kim J, et al. DTI Studio: resource program for diffusion tensor computation and fiber bundle tracking. Comput Methods Programs Biomed 2006;81:106-16

AJNR Am J Neuroradiol 34:785-90 Apr 2013 www.ajnr.org 
26. Nucifora PG, Verma R, Melhem ER, et al. Leftward asymmetry in relative fiber density of the arcuate fasciculus. Neuroreport 2005;16: 791-94

27. Vernooij MW, Smits M, Wielopolski PA, et al. Fiber density asymmetry of the arcuate fasciculus in relation to functional hemispheric language lateralization in both right- and left-handed healthy subjects: a combined fMRI and DTI study. Neuroimage 2007;35:1064-76

28. Neil JJ. Diffusion imaging concepts for clinicians. J Magn Reson Imaging 2008;27:1-7

29. Catani M, Mesulam M. The arcuate fasciculus and the disconnection theme in language and aphasia: history and current state. Cortex 2008;44:953-61

30. Bernal B, Ardila A. The role of the arcuate fasciculus in conduction aphasia. Brain 2009;132:2309-16

31. Shuren JE, Schefft BK, Yeh HS, et al. Repetition and the arcuate fasciculus. J Neurol 1995;242:596-98

32. Kim SH, Hong JH, You H, et al. The clinical application of the arcuate fasciculus for stroke patients with aphasia: a diffusion tensor tractography study. Neurorehabilitation 2011;29:305-10

33. Kertesz A, Harlock W, Coates R. Computer tomographic localization, lesion size, and prognosis in aphasia and nonverbal impairment. Brain Lang 1979;8:34-50

34. Marshall RC, Phillips DS. Prognosis for improved verbal communication in aphasic stroke patients. Arch Phys Med Rehabil 1983;64: 597-600

35. Laska AC, Hellblom A, Murray V, et al. Aphasia in acute stroke and relation to outcome. J Intern Med 2001;249:413-22
36. Fernandez B, Cardebat D, Demonet JF, et al. Functional MRI follow-up study of language processes in healthy subjects and during recovery in a case of aphasia. Stroke 2004;35:2171-76

37. Raboyeau G, De Boissezon X, Marie N, et al. Right hemisphere activation in recovery from aphasia: lesion effect or function recruitment? Neurology 2008;70:290-98

38. Saur D, Lange R, Baumgaertner A, et al. Dynamics of language reorganization after stroke. Brain 2006;129:1371-84

39. Kertesz A, McCabe P. Recovery patterns and prognosis in aphasia. Brain 1977;100 Pt 1:1-18

40. Ardila A. A review of conduction aphasia. Curr Neurol Neurosci Rep 2010;10:499-503

41. Rauschecker AM, Deutsch GK, Ben-Shachar M, et al. Reading impairment in a patient with missing arcuate fasciculus. Neuropsychologia 2009;47:180-94

42. Yang Q, Tress BM, Barber PA, et al. Serial study of apparent diffusion coefficient and anisotropy in patients with acute stroke. Stroke 1999;30:2382-90

43. Zelaya F, Flood N, Chalk JB, et al. An evaluation of the time dependence of the anisotropy of the water diffusion tensor in acute human ischemia. Magn Reson Imaging 1999;17:331-48

44. Parker GJ, Alexander DC. Probabilistic anatomical connectivity derived from the microscopic persistent angular structure of cerebral tissue. Philos Trans R Soc Lond B Biol Sci 2005;360:893-902

45. Huang H, Zhang J, van Zijl PC, et al. Analysis of noise effects on DTI-based tractography using the brute-force and multi-ROI approach. Magn Reson Med 2004;52:559-65 